\title{
Lateral-directional Attitude Under-actuated Control Strategy of Hypersonic Vehicle
}

\author{
Yike Niu ${ }^{1, a}$, Chunzhen Sun ${ }^{1, b}$, Yimin Huang ${ }^{1, c}$ and Xin Wang ${ }^{1, d}$ \\ ${ }^{1}$ School of Automation, Nanjing University of Aeronautics and Astronautics, Nanjing \\ aykn_nuaa@163.com, bsunchunzhen@nuaa.edu.cn \\ cymhuang@nuaa.edu.cn, dwangxin2001@nuaa.edu.cn
}

\begin{abstract}
Keywords: Hypersonic vehicle, Under-actuated, Cascade control.
Abstract. Research on lateral-directional attitude under-actuated control of the hypersonic vehicle with only the aileron, a new lateral-directional control strategy based on the idea of cascade was proposed, that can realize the roll angle control and guarantee the stabilization of the sideslip angle. Using the linear lateral-directional dynamic equations, the under-actuated control mechanism was analyzed. The rudder could produce sideslip angle, and the roll angle could be controlled by sideslip angle. With the concept of cascade control, a controller of two levels was designed that was series connection of the roll angle controller and sideslip angle controller. Results show that the design of the lateral-directional cascade control strategy can stabilize the system and the tacking performance of the roll angle satisfies the requirement.
\end{abstract}

\section{Introduction}

The degree of freedom is less than the dimension of under-actuated system [1]. The hypersonic vehicle becomes under-actuated system when there is a failure in actuator or the vehicle is designed to reduce the corresponding actuator. The control objective is difficult to achieve and the stability of the indirectly driven channel is not ensured under the limitation of the control surface. For the hypersonic vehicle with under-actuated characteristic, the under-actuated control technology is the guarantee of the attitude control and enhances the reliability of the system. Therefore, it has important theoretical significance and engineering application value for its further research.

The researches on under-actuated systems during the past decades are mainly on the optimal control method, motion planning method, partial feedback linearization method, energy method, variable dimension reduction method, cascade control design method, intelligent control method with its application in inverted pendulum system and aero-spacecraft but few in hypersonic vehicles under-actuated systems. To solve the problem of attitude control in the lateral-directional dynamic motion, inverse dynamics control based on output redefinition method is used to ensure the stability of system zero dynamic response [2,3]. Attitude control was designed based on inverse dynamics control method and the dynamic in the system was stabilized [4]. However, the inverse dynamics controller mentioned above may cause unstable in the internal system. The controller based on the singular perturbation theory cascaded with position and attitude was proposed to meet the performance requirements [5]. However, the parameter selection was very complicated and the cascade controller has not been used in hypersonic vehicles under-actuated systems before.

The methods described in the above literature are disadvantageous in that they are difficult to engineering realization and parameter tuning. In this paper, a new control strategy based on cascade under-actuated method is presented, which is clear and easy to be realized in engineering. In this paper, using cascade control theory, analyzes the mechanism of lateral-directional cascade control. Then the lateral-directional control is divided into two stages and two independent control strategies are designed. Finally, a lateral-directional cascade control strategy is presented. 


\section{Problem Description}

Assuming that the hypersonic vehicle is a rigid body, the linear lateral-directional dynamic equation can be written in the following form:

$$
\begin{aligned}
& \dot{\boldsymbol{x}}=\boldsymbol{A} \boldsymbol{x}+\boldsymbol{B u} \\
& \boldsymbol{y}=\boldsymbol{C} \boldsymbol{x}
\end{aligned}
$$

The states $\boldsymbol{x}$, the outputs $\boldsymbol{y}$ and the controls $\boldsymbol{u}$ are expressed as:

$$
\begin{aligned}
\boldsymbol{x} & =[\beta, p, r, \phi]^{\mathrm{T}} \\
\boldsymbol{y} & =[\beta, p, r, \phi]^{\mathrm{T}} \\
\boldsymbol{u} & =\delta_{\boldsymbol{a}}
\end{aligned}
$$

where $\beta$ is the sideslip angle, $p$ is roll angular rate, $r$ is yaw angular rate, $\phi$ is the roll angle and $\delta_{a}$ is the aileron.

The Dutch-roll motion is unstable. If there exists sideslip angle perturbation, the sideslip angle will diverge during the flight without sideslip angle control, which can also lead to the divergence of roll angle. Therefore, to ensure the convergence of the sideslip angle is very important for roll angle control. However, the sideslip angle and roll angle control must be realized expected roll angle and ensured sideslip angle bounded by saturated aileron.

In order to satisfy the requirement of lateral-directional control, the constraint condition can be denoted as:

$$
\left\{\begin{array}{l}
\operatorname{Re}|s \boldsymbol{I}-\boldsymbol{A}|_{s i}<0,(i=1,2 \ldots) \\
\beta_{\text {min }} \leq \beta \leq \beta_{\max } \\
\phi=\phi_{\text {cmd }}
\end{array}\right.
$$

where $\operatorname{Re}|s \boldsymbol{I}-\boldsymbol{A}|_{s i}$ is the real part of lateral-directional characteristic root.

\section{Lateral-directional Control Mechanism}

The system matrices $A$ and $B$ are:

$$
\boldsymbol{A}=\left[\begin{array}{cccc}
Y_{\beta}^{\prime} & Y_{\mathrm{p}}^{\prime} & Y_{r}^{\prime} & g_{2}^{\phi^{\prime}} \\
\overline{L_{\beta}} & \overline{L_{p}} & \overline{L_{r}} & 0 \\
N_{\beta}^{\prime} & N_{p}^{\prime} & N_{r}^{\prime} & 0 \\
0 & 1 & 0 & \phi_{\phi}
\end{array}\right], \boldsymbol{B}=\left[\begin{array}{c}
Y_{\delta_{a}} \\
\bar{L}_{\delta_{a}} \\
N_{\delta_{a}}^{\prime} \\
0
\end{array}\right]
$$

where $D_{i}^{\prime}\left(D^{\prime}=Y^{\prime}, \bar{L}, N^{\prime}, i=\beta, p, r\right)$ is the derivative of $D^{\prime}$ to $i$. The second-order equations for the lateral dynamics can be derived as:

$$
\begin{aligned}
& \Delta \ddot{\beta}=Y_{\beta}^{\prime} \Delta \dot{\beta}+\left(Y_{p}^{\prime} \bar{L}_{\beta}+Y_{r}^{\prime} N_{\beta}^{\prime}\right) \Delta \beta+D \Delta p+g_{2}^{\phi^{\prime}} \phi_{\phi} \Delta \phi \\
& \Delta \ddot{\phi}=\left(\bar{L}_{\beta}+N_{\beta}^{\prime} \phi_{r}\right) \Delta \beta+\left(\left(\bar{L}_{p}+\bar{L}_{r} \frac{N_{\beta}^{\prime}}{\bar{L}_{\beta}}\right)+\phi_{r}\left(N_{p}^{\prime}+N_{r}^{\prime} \frac{N_{\beta}^{\prime}}{\bar{L}_{\beta}}\right) \Delta p\right.
\end{aligned}
$$

where $D$ can be expressed as:

$$
D=Y_{p}^{\prime}\left(\bar{L}_{p}+\bar{L}_{r} \frac{N_{\beta}^{\prime}}{\bar{L}_{\beta}}\right)+Y_{r}^{\prime}\left(N_{p}^{\prime}+N_{r}^{\prime} \frac{N_{\beta}^{\prime}}{\bar{L}_{\beta}}\right)+g_{2}^{\phi^{\prime}}\left(1+\phi_{r} \frac{N_{\beta}^{\prime}}{\bar{L}_{\beta}}\right)
$$

Using Eq. 5, the transfer function from the sideslip angle to the rolling angle is obtained as: 
$G(s)=\frac{\varphi(s)}{\beta(s)}=\frac{E}{D} \frac{s^{2}-Y_{\beta}^{\prime} s-\left[\left(Y_{p}^{\prime} \bar{L}_{\beta}+Y_{r}^{\prime} N_{\beta}^{\prime}\right)-\frac{D}{E}\left(\bar{L}_{\beta}+\phi_{r} N_{\beta}^{\prime}\right)\right]}{s^{2}-\phi_{\phi} s+g_{2}^{\phi^{\prime}} \phi_{\phi} D}$

where $E$ and $F$ can be denoted in the following form:

$E=\left(\bar{L}_{p}+\bar{L}_{r} \frac{N_{\beta}^{\prime}}{\bar{L}_{\beta}}\right)+\left(N_{p}^{\prime}+N_{r}^{\prime} \frac{N_{\beta}^{\prime}}{\bar{L}_{\beta}}\right) \phi_{\mathrm{r}}, F=N_{\beta}^{\prime}\left[\left(Y_{p}^{\prime}-\frac{D}{E}\right) \frac{\bar{L}_{\beta}}{N_{\beta}^{\prime}}+\left(Y_{r}^{\prime}-\frac{D}{E} \phi_{r}\right)\right]$

Considering that $\phi_{\phi}$ and $\mathrm{g}_{2}^{\phi^{\prime}}$ are small, $\mathrm{g}_{2}^{\phi^{\prime}} \phi_{\phi} D$ can be ignored. The frequency response of the system $G(s)$ can be denoted as:

$$
|G(j \omega)|=\frac{E}{D} \frac{\sqrt{\left[Y_{\beta}^{\prime} \omega \phi_{\phi}+\left(F+\omega^{2}\right) \omega\right]^{2}+\left[Y_{\beta}^{\prime} \omega^{2}-\phi_{\phi}\left(F+\omega^{2}\right)\right]^{2}}}{\omega\left(\omega^{2}+\phi_{\phi}^{2}\right)}, \angle G(j \omega)=-90^{\circ}+\arctan \frac{Y_{\beta}^{\prime} \omega}{F+\omega^{2}}-\arctan \frac{\omega}{\phi_{\phi}}
$$

From the Eq. 6 and Eq. 8, if $\left|\bar{L}_{\beta} / N_{\beta}^{\prime}\right| \gg 1,|E / D|$ satisfies:

$$
|E / D| \rightarrow\left(\bar{L}_{p}+N_{p}^{\prime} \phi_{\mathrm{r}}\right) /\left(Y_{p}^{\prime} \bar{L}_{p}+Y_{r}^{\prime} N_{p}^{\prime}+g_{2}^{\phi^{\prime}}\right)
$$

Considering that $\phi_{\phi}$ is small and can be ignored, if $\left|\bar{L}_{\beta} / N_{\beta}^{\prime}\right| \gg 1$, then $|G(j \omega)| \gg 1$. The $\left|\bar{L}_{\beta} / N_{\beta}^{\prime}\right|$ of the vehicle is more than 15 , so one degree of sideslip angle can produce larger roll angle, which indicates that the roll angle can be controlled by sideslip angle. The bode diagram of the system transfer function from sideslip angle to roll angle is shown in the Fig. 1.

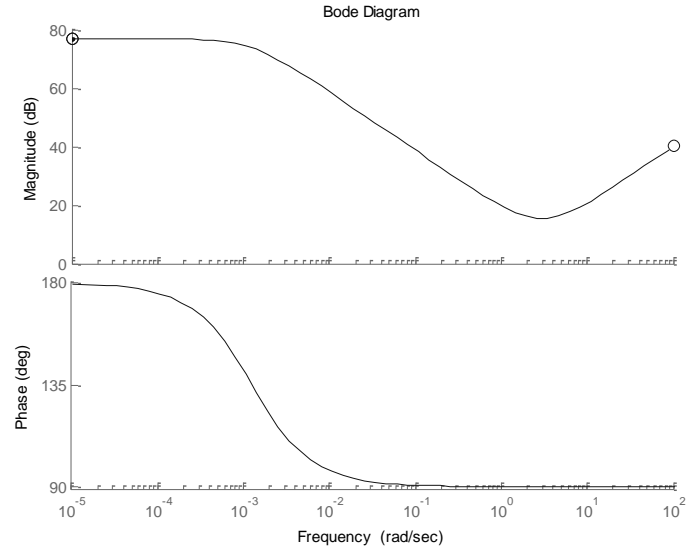

Fig. 1. The sideslip angle to roll angle Bode diagram

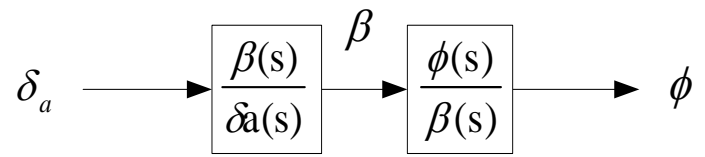

Fig. 2. The system structure diagram from aileron to roll angle

From Fig. 1 and the Eq. 9 can be shown that the sideslip angle phase angle lags behind 90 degrees compared with the roll angle when $\left|\bar{L}_{\beta} / N_{\beta}^{\prime}\right|$ is large.

According to the Eq. 4, the transfer function of aileron to sideslip angle is expressed as:

$$
\frac{\beta(s)}{\delta_{\mathrm{a}}(s)}=\frac{Y_{\delta \mathrm{a}} s^{2}+\left(\bar{L}_{\delta a} Y_{p}^{\prime}+N_{\delta \mathrm{a}}^{\prime} Y_{r}^{\prime}-Y_{\delta \mathrm{a}}\left(N_{r}^{\prime}+\bar{L}_{p}\right)\right) s+a_{0}}{s^{3}+\left(N_{r}^{\prime}+Y_{\beta}^{\prime}-\bar{L}_{p}+\phi_{\phi}\right) s^{2}+b_{1} s+b_{0}}
$$

Where $a_{0}, b_{0}, b_{1}$ are denoted as:

$$
\begin{aligned}
& a_{0}=Y_{\delta \mathrm{a}}\left(\bar{L}_{p} N_{r}^{\prime}-\bar{L}_{r} N_{p}^{\prime}\right)+\bar{L}_{\delta a}\left(Y_{r}^{\prime} N_{p}^{\prime}-Y_{p}^{\prime} N_{r}^{\prime}\right)+N_{\delta \mathrm{a}}^{\prime}\left(Y_{p}^{\prime} \bar{L}_{r}-Y_{r}^{\prime} \bar{L}_{p}\right) \\
& b_{0}=Y_{\beta}^{\prime}\left(\bar{L}_{\mathrm{r}} N_{p}^{\prime}-\bar{L}_{p} N_{r}^{\prime}\right)+\bar{L}_{\beta}\left(Y_{p}^{\prime} N_{r}^{\prime}-Y_{r}^{\prime} N_{p}^{\prime}\right)+N_{\beta}^{\prime}\left(Y_{r}^{\prime} \bar{L}_{p}-Y_{p}^{\prime} \bar{L}_{r}\right), \quad b_{1}=\bar{L}_{p} Y_{\beta}^{\prime}-\bar{L}_{\beta} Y_{p}^{\prime}+N_{r}^{\prime} Y_{\beta}^{\prime}-N_{\beta}^{\prime} Y_{r}^{\prime}+\vec{L}_{p} N_{r}^{\prime}-\vec{L}_{r} N_{p}^{\prime}
\end{aligned}
$$


When the derivative $N_{\delta \mathrm{a}}^{\prime}$ and $Y_{r}^{\prime}$ are large, the aileron can produce coupling torque. With this relationship, the system structure diagram from aileron to roll angle is shown in the Fig. 2 . When $\left|\bar{L}_{\beta} / N_{\beta}^{\prime}\right|$ is relatively large, the sideslip angle system and roll angle system can be concatenated. Aileron is used to produce the coupling torque and roll angle is controlled by sideslip angle.

\section{Under-actuated Control Strategy}

Through the analysis of the previous section, lateral-directional control can be divided into the control of sideslip angle and roll angle. Using the idea of cascade control, the control of the sideslip angle is regarded as the inner lateral-directional control, and the outer loop is roll angle. The structure diagram of the cascade control strategy is shown in Fig. 3.

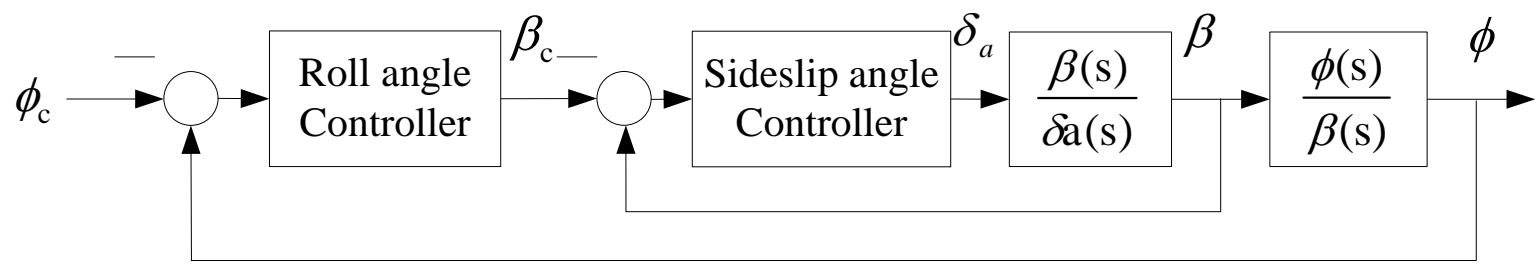

Fig. 3 The structure diagram of the cascade control strategy

Sideslip Angle Control Strategy. For the under-actuated spacecraft yaw instability, in critical stable state, and stable but higher performance requirements of mobile, Dutch-roll is likely to be unstable. The characteristic parameter damping ratio and frequency can improve the stability characteristics of Dutch-roll. The two order system of the sideslip angle has the same characteristic with Dutch-roll mode.

The sideslip angle and sideslip angle acceleration are feedback to the aileron, and the characteristic parameters of the sideslip angle of two order closed-loop system respectively are expressed as:

$$
\begin{aligned}
& \omega_{\mathrm{n}}^{2}=N_{r}^{\prime} Y_{\beta}^{\prime}+\left(N_{\beta}^{\prime}+k_{a}^{\beta} N_{\delta a}^{\prime}\right) \\
& \xi=-\left(N_{r}^{\prime}+Y_{\beta}^{\prime}\right) / 2 \omega_{n} \\
& \omega_{\mathrm{n}}^{2}=N_{r}^{\prime} Y_{\beta}^{\prime}+N_{\beta}^{\prime} \\
& \xi=-\left(N_{r}^{\prime}-k_{a}^{\dot{\beta}} N_{\delta_{a}}+Y_{\beta}^{\prime}\right) / 2 \omega_{n}
\end{aligned}
$$

Eq. 13 and Eq. 14 shows that the sideslip angle feedback can improve the frequency characteristics effectively and the sideslip angle acceleration feedback can improve the dynamic quality of sideslip angle. Thus, the control strategy of sideslip angle is in the following form:

$$
\delta_{a}=k_{a}^{\beta}\left(\beta-\beta_{\mathrm{c}}\right)+k_{a}^{\dot{\beta}} \dot{\beta}
$$

where $\beta_{c}$ is the command of the sideslip angle.

Roll Angle Control Strategy. According to the above analysis, the roll angle can be controlled by the sideslip angle. The control system the sideslip angle is regard as an object and the control structure diagram of the roll angle is shown in Fig. 4. 


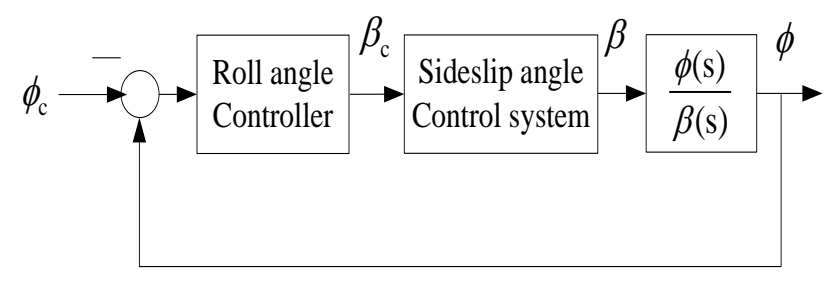

Fig. 4. The control structure diagram of the roll angle

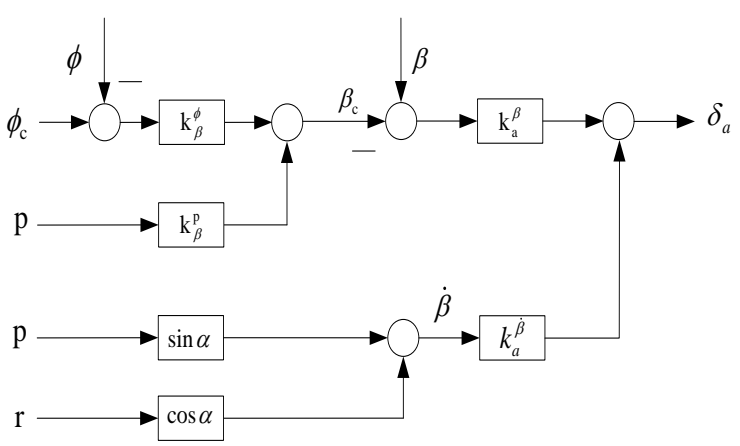

Fig. 5. The structure diagram of the lateral-directional control law

The roll angle feedback can improve response speed of the system, and roll angular rate feedback can improve the damping characteristics of the rolling movement. Therefore, the control strategy of the roll angle can be expressed as:

$$
\beta_{c}=k_{\beta}^{\phi}\left(\phi-\phi_{c}\right)+k_{\beta}^{p} p
$$

According to the design of control strategy in Eq. 15 and Eq. 16, the structure diagram of the lateral-directional control law can be shown in Fig. 5. The state feedback is adopted by the control of the sideslip angle to ensure the state stability of the sideslip angle. The control method of output feedback is adopted to realize the tracking control of roll angle. The sideslip angular rate can inhibit the movement coupling and play a lateral-directional coordinating role that can change the dynamic regulation of lateral-directional angular velocity.

\section{Simulation Results}

In order to verify the effectiveness and correctness of the designed lateral-directional cascade control strategy, the six degree of freedom nonlinear simulation platform is built by using Maltab/Simulink. The initial conditions for nonlinear simulation: height is $55 \mathrm{~km}$, angle of attack is 22.5 degrees, the roll angle is 3.3 degrees, and the sideslip angle is 0 degree. The expected roll angle commands are 45 degrees, -35 degrees and 0 degree. The sideslip angle of the absolute value is not greater than 3 degrees. The rudder range is $0 \sim 15$ degrees. The curve of roll angle and sideslip angle is shown in Fig. 6. The curve of aileron is shown in Fig. 7.

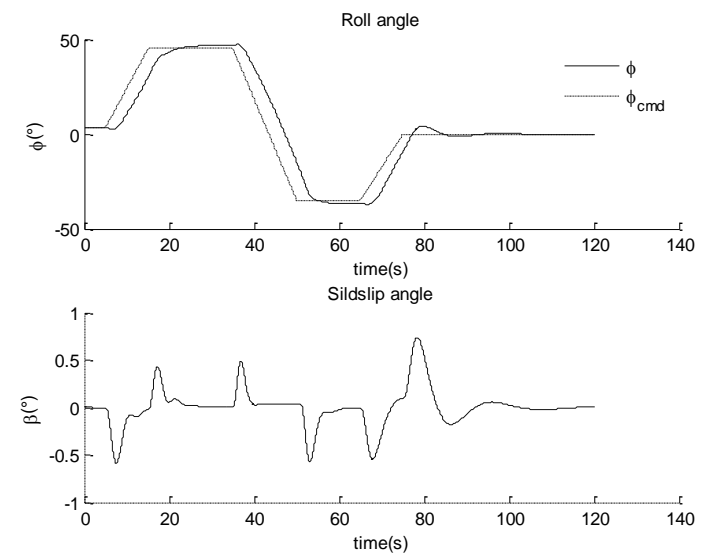

Fig. 6. The curve of roll angle and sideslip angle

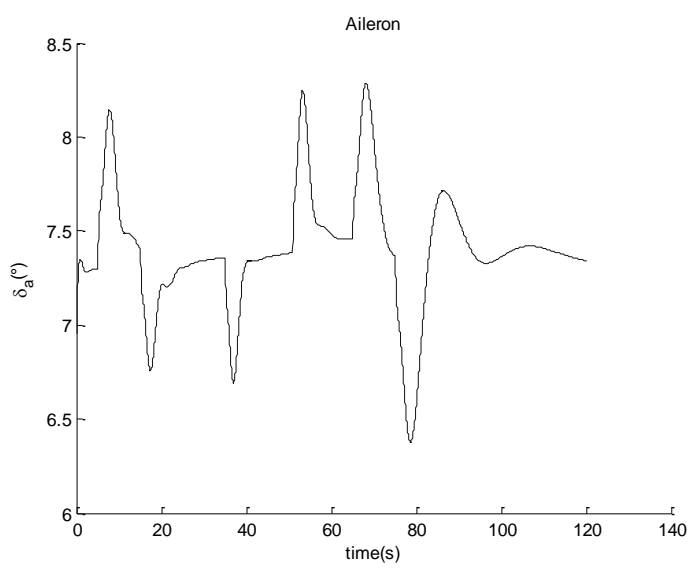

Fig. 7. The curve of aileron

Fig. 6 shows that the roll angle can be tracked on the roll angle command with the designed control strategy of under-actuated cascade, but there will be some delay. When roll angle is controlled by sideslip angle, there will be a certain phase delay, which is consistent with the previous results of the mechanism analysis. The steady-state error of roll angle is 2 degrees, meeting the requirements. Although the maximal deviation of sideslip angle is 1 degree, it quickly converges to zero. Fig. 7 shows that the demand of the aileron is almost 7.5 degrees and the margin is large. It is showed that the design of lateral-directional control strategy of under-actuated cascade is effective. 


\section{Conclusions}

In this paper, a new lateral-directional control strategy based on the idea of cascade for hypersonic vehicle is proposed. The main contributions are as follows:

1. The under-actuated control mechanism was analyzed. The control surface could produce sideslip angle, and the roll angle could be controlled by sideslip angle, which is different from the traditional method.

2. With the concept of cascade control, the lateral-directional control is divided into sideslip angle control and roll angle control two stages and then design the control strategy for every stage.

3. The simulation results show that the under-actuated control method is effective for the lateral-directional attitude control of the large $\left|\bar{L}_{\beta} / N_{\beta}^{\prime}\right|$ hypersonic vehicle without external wind disturbance.

\section{Acknowledgements}

This work was financially supported by the Fundamental Research Funds for the Central Universities (No.2015038).

\section{References}

[1] NingSun, Yongchun Fang. CAAI Transactions on Intelligent Systems, 6(3): 200-207, "In Chinese". ( 2011)

[2] Linan Shi, Ran Zhang, Zhaoying Li, etal. Journal of Beijing University of Aeronautics and Astronautics, 40(9): 1291-1298, "In Chinese". (2014)

[3] Elmar M. Wallner* and Kaus H. AIAA. 5-8 August, Monterey, California. (2002)

[4] Ito D, Georgie J, Valasek J. NASA/TP-2002-210-771. (2002)

[5] Sylvain Bertrand, Nicolas Guenard, Tarek Hamel: Control Engineering Practice. Vol.19, no.10, 1099-1108. ( 2011)

[6] Daozhe Song, Yunhai Geng, Tao Yi. Journal of Astronautics, 37(6): 729-736, "In Chinese". (2016)

[7] Linan Shi, Huifeng Li, Ran Zhang. Journal of Beijing University of Aeronautics and Astronautics, 40(9): 1000-1005, "In Chinese". (2014)

[8] Xiaoyu Zhang, Zicai Wang. Journal of Astronautics. 28(3): 531-534. (2007) 